\title{
Algebraic error analysis of collinear feature points for camera parameter estimation ${ }^{\text {मे }}$
}

\author{
Onay Urfalioglu ${ }^{\mathrm{a}, *}$, Thorsten Thormählen ${ }^{\mathrm{b}}$, Hellward Broszio ${ }^{\mathrm{c}}$, Patrick Mikulastik ${ }^{\mathrm{c}}$, A. Enis Cetin ${ }^{\mathrm{a}}$ \\ a Department of Electrical and Electronics Engineering, Bilkent University, Ankara, Turkey \\ ${ }^{\mathrm{b}}$ Max Planck Institute for Computer Science, Saarbrücken, Germany \\ ' Information Technology Laboratory, Leibniz University Hannover, Germany
}

\section{A R T I C L E I N F O}

Article history:

Received 6 December 2008

Accepted 23 December 2010

Available online 4 January 2011

\section{Keywords:}

Collinear

Covariance propagation

Error analysis

Cramer-Rao bounds

ML-estimation

Camera parameter estimation

\begin{abstract}
A B S T R A C T
In general, feature points and camera parameters can only be estimated with limited accuracy due to noisy images. In case of collinear feature points, it is possible to benefit from this geometrical regularity by correcting the feature points to lie on the supporting estimated straight line, yielding increased accuracy of the estimated camera parameters. However, regarding Maximum-Likelihood (ML) estimation, this procedure is incomplete and suboptimal. An optimal solution must also determine the error covariance of corrected features. In this paper, a complete theoretical covariance propagation analysis starting from the error of the feature points up to the error of the estimated camera parameters is performed. Additionally, corresponding Fisher Information Matrices are determined and fundamental relationships between the number and distance of collinear points and corresponding error variances are revealed algebraically. To demonstrate the impact of collinearity, experiments are conducted with covariance propagation analyses, showing significant reduction of the error variances of the estimated parameters.
\end{abstract}

(c) 2010 Elsevier Inc. All rights reserved.

\section{Introduction}

In feature point based structure-from-motion (SFM) methods [1-11], the accuracy of the estimated camera parameters depends on the accuracy of the detected features. The knowledge of the probability density function of feature point positions enables the use of Maximum-Likelihood (ML) estimation theory [12]. Based on ML-theory, it is also possible to determine the expected error covariances of the estimated camera parameters [12]. In [13], Bartoli et al. propose the utilization of collinear features when appropriate. In synthetic experiments, it is shown that the utilization of collinear features leads to smaller covariances of estimated camera parameter errors. In [14], a simplified error covariance propagation analysis for collinear features is presented for SFM, but the analysis is not based on optimal ML-estimation, therefore a fully ML-based theoretical analysis of covariance propagation is missing.

To exploit collinearity, this paper determines an ML-estimate of a (straight) line which is supported by some feature points. The feature point positions are then corrected by projecting them onto the estimated line. The estimation of camera parameters is based

This work was partly published in IEEE Proceedings of the Canadian Conference on Computer and Robot Vision 2006 (CRV2006).

* Corresponding author.

E-mail addresses: onay@ee.bilkent.edu.tr (O. Urfalioglu), thormae@mpi-inf. mpg.de (T. Thormählen), broszio@tnt.uni-hannover.de (H. Broszio), mikulast@tnt. uni-hannover.de (P. Mikulastik), cetin@bilkent.edu.tr (A.E. Cetin). on the corrected feature points. Thereby, it is proven that the resulting feature points have smaller error covariances resulting in higher accuracy of the estimated camera parameters.

Several proposed methods exist for estimating lines and determining the error covariances of the line parameters and corresponding Cramer-Rao lower bounds analytically [15-17]. In this paper, the covariance and the Cramer-Rao bound determination of line parameters is reviewed. The main contribution is an analysis of corrected point positions including the determination of the error covariances and the Cramer-Rao bounds, which depend both on the uncertainty of the line as well as on the uncertainty of the selected point to be corrected. Additionally, the Cramer-Rao terms are further analyzed to derive fundamental relationships between the number of supporting points, the line parameter accuracy and the accuracy of the corrected features.

The focus is on camera parameter estimation, so a complete theoretical analysis starting from the error of the feature points up to the error of the estimated camera parameters is presented.

In Section 2, the ML-estimation of a line in an image is presented. In Section 3, the error covariance propagation for the corrected feature points is analytically derived. Section 4 describes the calculation of the Fisher Information Matrix and the Cramer-Rao bounds for the expected error covariances of corrected feature points. Section 5 describes briefly the propagation of the error covariances up to the camera parameters followed by Section 6 in which the usefulness of collinearity is experimentally demonstrated. 


\section{Maximum-Likelihood estimation of line parameters}

A set of feature points is given, which is supposed to lie on a straight line. However, their locations are erroneous so they actually are not located on the line exactly.

The goal is to determine the line parameters by processing information given by the feature points. A 2D-line $\boldsymbol{l}$ can be described by the Hessian parameterization. A point $\boldsymbol{x}$ lies on a line if

$\boldsymbol{n}^{\top}(\boldsymbol{a}-\boldsymbol{x})=0$,

where

$\boldsymbol{n}=(\cos (\phi), \sin (\phi))^{\top}$,

is the normal vector and $\boldsymbol{a}$ is the base. By defining the homogeneous point

$\boldsymbol{x}=(x, y, 1)^{\top}$,

and

$\boldsymbol{l}=(\cos (\phi), \sin (\phi),-\rho)^{\top}$,

the homogeneous parameterization of the line is obtained, satisfying

$\boldsymbol{I}^{\top} \boldsymbol{x}=0$.

It is assumed that the probability density function (PDF) describing the uncertainty of the feature points is arbitrary Gaussian and the covariances are known. The error covariances can be determined by analyzing the feature tracking method, e.g. for the KLT tracking method $[18,19]$, the error analysis for the position of the detected features can be found in [20]. In order to take maximum benefit from the knowledge of the PDF, parameters can be determined using ML-estimation. The position error of a feature point $\boldsymbol{x}$ has the PDF

$p(\boldsymbol{x} \mid \overline{\boldsymbol{x}})=\frac{e^{\left[-\frac{1}{2}(\boldsymbol{x}-\overline{\boldsymbol{x}})^{\top} \mathrm{C}^{-1}(\boldsymbol{x}-\overline{\boldsymbol{x}})\right]}}{2 \pi \sqrt{\operatorname{det}(\mathrm{C})}}$,

where $\boldsymbol{x}$ is the measured point, $\overline{\boldsymbol{x}}$ is the true point and $\mathrm{C}$ is the covariance matrix. It is assumed that the position errors of feature points are statistically independent. Let

$\boldsymbol{z}=\left(\boldsymbol{x}^{(1)^{\top}}, \ldots, \boldsymbol{x}^{(M)^{\top}}\right)^{\top}$,

be the vector of all points belonging to the estimated line. The task is to estimate the corresponding points $\hat{\boldsymbol{x}}^{(i)}$ on the line, so that the likelihood $L$

$L=\prod_{i}^{M} p\left(\boldsymbol{x}^{(i)} \mid \hat{\boldsymbol{x}}^{(i)}\right)$,

is maximized.

For a specific line $(\phi, \rho)$, the estimated point $\hat{\boldsymbol{x}}^{(i)}$ can be determined directly. For ML-estimation, the maximization of the Likelihood is equivalent to

$\hat{\boldsymbol{x}}^{(i)}=\arg \max _{\tilde{\boldsymbol{x}}^{(i)}} p\left(\boldsymbol{x}^{(i)} \mid \tilde{\boldsymbol{x}}^{(i)}\right)$,

which yields

$\hat{\boldsymbol{x}}^{(i)}=\arg \max _{\tilde{\boldsymbol{x}}^{(i)}} \frac{e^{\left[-\frac{1}{2}\left(\boldsymbol{x}^{(i)}-\tilde{\boldsymbol{x}}^{(i)}\right)^{\top} \mathrm{C}^{(i)}{ }^{-1}\left(\boldsymbol{x}^{(i)}-\tilde{\boldsymbol{x}}^{(i)}\right)\right]}}{2 \pi \sqrt{\operatorname{det}\left(C^{(i)}\right)}}$,

with the constraint that the point $\hat{\boldsymbol{x}}$ must lie on the line $(\phi, \rho)$. This constraint is expressed by

$\hat{\boldsymbol{x}}^{(i)}=\left(\begin{array}{c}\rho \cos (\phi) \\ \rho \sin (\phi)\end{array}\right)+\lambda^{(i)}\left(\begin{array}{c}\sin (\phi) \\ -\cos (\phi)\end{array}\right)=\boldsymbol{a}+\lambda^{(i)} \boldsymbol{b}$ where $\lambda^{(i)}$ is a scalar, $\boldsymbol{a}$ is a supporting vector and $\boldsymbol{b}$ is the direction vector. With this constraint and some additional simplifications, the condition (10) becomes

$\hat{\lambda}^{(i)}=\arg \min _{\lambda^{(i)}}\left(\boldsymbol{x}^{(i)}-\boldsymbol{a}-\lambda^{(i)} \boldsymbol{b}\right)^{\top} \mathrm{C}^{(i)}{ }^{-1}\left(\boldsymbol{x}^{(i)}-\boldsymbol{a}-\lambda^{(i)} \boldsymbol{b}\right)$.

In order to minimize Eq. (12), following condition must hold

$\left.\frac{\partial}{\partial \lambda^{(i)}}\left(\boldsymbol{x}^{(i)}-\boldsymbol{a}-\lambda^{(i)} \boldsymbol{b}\right)^{\top} \mathrm{C}^{(i)}\left(\boldsymbol{x}^{(i)}-\boldsymbol{a}-\lambda^{(i)} \boldsymbol{b}\right)\right|_{\lambda^{(i)}=\hat{\lambda}^{(i)}}=0$

which yields

$\hat{\lambda}^{(i)}=\frac{\left(\boldsymbol{x}^{(i)}-\boldsymbol{a}\right)^{\top} \mathrm{C}^{(i)^{-1} \boldsymbol{b}}}{\boldsymbol{b}^{\top} \mathrm{C}^{(i)}{ }^{-1} \boldsymbol{b}}$.

Apparently, $\lambda^{(i)}$ is a function of $\left(\phi, \rho, \boldsymbol{x}^{(i)}\right): \lambda^{(i)}\left(\phi, \rho, \boldsymbol{x}^{(i)}\right)$, and $\hat{\boldsymbol{x}}^{(i)}$ is a function of $\lambda^{(i)}: \hat{\boldsymbol{x}}^{(i)}\left(\lambda^{(i)}\left(\phi, \rho, \boldsymbol{x}^{(i)}\right)\right)$. Finally, the following cost function is used for the estimation of the line parameters $(\phi, \rho)$

$(\hat{\phi}, \hat{\rho})=\arg \min _{(\phi, \rho)} \sum_{i}^{M}\left[\boldsymbol{x}^{(i)}-\hat{\boldsymbol{x}}^{(i)}\left(\hat{\lambda}^{(i)}\right)\right]^{\top} C^{(i)}{ }^{-1}\left[\boldsymbol{x}^{(i)}-\hat{\boldsymbol{x}}^{(i)}\left(\hat{\lambda}^{(i)}\right)\right]$.

The cost function in (15) can be minimized by iterative optimization methods.

\section{Propagation of error covariance}

In order to determine the impact of the collinearity on the accuracy of the camera parameters, error covariances are propagated from the feature points up to the camera parameters. The propagation is started from the detected points up to the line defined in Section 3.1 and continued from the line up to the corrected/projected points in Section 3.2.

\subsection{Error covariance of line parameters}

The cost function (15) has the form

$f(\hat{\phi}(\boldsymbol{z}), \hat{\rho}(\boldsymbol{z}) ; \boldsymbol{z})=\sum_{i=1}^{M} \boldsymbol{d}^{(i)^{\top}} \mathrm{C}^{(i)^{-1}} \boldsymbol{d}^{(i)}$,

with

$\boldsymbol{d}^{(i)}=\boldsymbol{x}^{(i)}-\hat{\boldsymbol{x}}^{(i)}$.

A necessary condition is that the gradient becomes zero

$\boldsymbol{h}=\operatorname{grad} f=\left.\frac{d}{d(\phi, \rho)} f(\phi(\boldsymbol{z}), \rho(\boldsymbol{z}) ; \boldsymbol{z})\right|_{\phi=\hat{\phi}, \rho=\hat{\rho}} \stackrel{!}{=} \mathbf{0}$.

It is not possible to resolve this equation for $(\hat{\phi}, \hat{\rho})$ algebraically in a trivial way. This means that there is no closed form solution for a mapping $\mathbf{g}$ with

$\mathbb{R}^{2 M} \rightarrow \mathbb{R}^{2}:(\hat{\phi}, \hat{\rho})^{\top}=\mathbf{g}(\boldsymbol{z})$,

where $(\hat{\phi}, \hat{\rho}) \in \mathbb{R}^{2}$ and $\boldsymbol{z} \in \mathbb{R}^{2 M}$. On the other hand, the implicitly defined function $\boldsymbol{h}(\mathbf{g}(\boldsymbol{z}), \boldsymbol{z}) \stackrel{!}{=} \mathbf{0}$ enables the calculation of the Jacobian $\frac{d g}{d z}$ by utilizing the theorem about implicit functions in order to determine the first order approximation of the desired function $\mathbf{g}$

$\left(\begin{array}{ll}\partial_{x^{(i)}} g_{1} & \partial_{y^{(i)}} g_{1} \\ \partial_{x^{(i)}} g_{2} & \partial_{y^{(i)}} g_{2}\end{array}\right)=-\left(\begin{array}{cc}\partial_{\phi} h_{1} & \partial_{\rho} h_{1} \\ \partial_{\phi} h_{2} & \partial_{\rho} h_{2}\end{array}\right)^{-1}\left(\begin{array}{cc}\partial_{x^{(i)}} h_{1} & \partial_{y^{(i)}} h_{1} \\ \partial_{x^{(i)}} h_{2} & \partial_{y^{(i)}} h_{2}\end{array}\right)$

where $\partial_{a} \equiv \frac{\partial}{\partial a}$ and $\partial_{a, b} \equiv \frac{\partial^{2}}{\partial_{a} \partial_{b}}$. This yields

$$
\begin{array}{r}
\partial_{z} \boldsymbol{g}=-\left(\partial_{\boldsymbol{g}} \boldsymbol{h}\right)^{-1} \partial_{z} \boldsymbol{h} \\
=-\left(\partial_{\phi, \rho} \boldsymbol{h}\right)^{-1} \partial_{z} \boldsymbol{h} .
\end{array}
$$


The linearized function is

$$
\boldsymbol{g}(\boldsymbol{z}+\mathbf{e}) \approx\left(\begin{array}{c}
\hat{\phi} \\
\hat{\rho}
\end{array}\right)+\underbrace{\left(\begin{array}{lllll}
\partial_{x^{(1)}} g_{1} & \partial_{y^{(1)}} g_{1} & \cdots & \partial_{x^{(M)}} g_{1} & \partial_{y^{(M)}} g_{1} \\
\partial_{x^{(1)}} g_{2} & \partial_{y^{(1)}} g_{2} & \cdots & \partial_{x^{(M)}} g_{2} & \partial_{y^{(M)}} g_{2}
\end{array}\right)}_{\mathrm{A}} \mathbf{e}
$$

$\mathbf{g}(\boldsymbol{z}+\mathbf{e}) \approx(\hat{\phi}, \hat{\rho})^{\top}+\mathrm{Ae}$.

After determining the first order approximation the error covariance of the line parameters can be specified

$$
\operatorname{cov}(\hat{\phi}, \hat{\rho})=\Lambda=\mathrm{A}\left(\begin{array}{ccccc}
\mathrm{C}_{11}^{(1)} & \mathrm{C}_{12}^{(1)} & & & \\
\mathrm{C}_{21}^{(1)} & \mathrm{C}_{22}^{(1)} & & & \\
& & \ddots & & \\
& & & \mathrm{C}_{11}^{(M)} & \mathrm{C}_{12}^{(M)} \\
& & & \mathrm{C}_{21}^{(M)} & \mathrm{C}_{22}^{(M)}
\end{array}\right) \mathrm{A}^{\top} .
$$

\subsection{Error covariance of corrected point position}

In this section, the position error covariance of a corrected point is determined. Given line parameters, let $\boldsymbol{P}$ the function which projects a point onto the line. This is also referred to as correcting a point. This function is determined by Eqs. (11) and (14)

$$
\begin{aligned}
\hat{\boldsymbol{x}}^{(i)} & =\left.\boldsymbol{P}\left(\phi, \rho, \boldsymbol{x}^{(i)}\right)\right|_{\phi=\hat{\phi}, \rho=\hat{\rho}} \\
& =\boldsymbol{a}(\phi, \rho)+\left.\lambda^{(i)}\left(\phi, \rho, \boldsymbol{x}^{(i)}\right) \boldsymbol{b}(\phi, \rho)\right|_{\phi=\hat{\phi}, \rho=\hat{\rho}} .
\end{aligned}
$$

To calculate the error, the first order Taylor series of $\boldsymbol{P}$ yields

$$
\boldsymbol{P}\left(\left(\hat{\phi}, \hat{\rho}, x^{(i)}, \boldsymbol{y}^{(i)}\right)^{\top}+\boldsymbol{\delta}\right) \approx\left(\begin{array}{c}
\hat{\boldsymbol{x}}^{(i)} \\
\hat{y}^{(i)}
\end{array}\right)+\underbrace{\left(\begin{array}{cccc}
\partial_{\phi} \boldsymbol{P}_{1} & \partial_{\rho} \boldsymbol{P}_{1} & \partial_{x^{(i)}} \boldsymbol{P}_{1} & \partial_{y^{(i)}} \boldsymbol{P}_{1} \\
\partial_{\phi} \boldsymbol{P}_{2} & \partial_{\rho} \boldsymbol{P}_{2} & \partial_{x^{(i)}} \boldsymbol{P}_{2} & \partial_{y^{(i)}} \boldsymbol{P}_{2}
\end{array}\right)}_{\mathrm{B}^{(i)}\left(\phi, \rho, x^{(i)}, y^{(i)}\right)} \boldsymbol{\delta},
$$

where

$$
\boldsymbol{\delta}=\left(\begin{array}{c}
\delta \phi \\
\delta \rho \\
\delta \boldsymbol{x}^{(i)} \\
\delta \boldsymbol{y}^{(i)}
\end{array}\right)
$$
by

The error covariance of a projected point can be approximated

$$
\widehat{\mathrm{C}}^{(i)}=\operatorname{cov}\left(\hat{\boldsymbol{x}}^{(i)}\right)=\mathrm{B}^{(i)}\left(\begin{array}{cc}
\Lambda & 0 \\
0 & \mathrm{C}^{(i)}
\end{array}\right) \mathrm{B}^{(i)^{\top}},
$$

where $\mathrm{B}^{(i)}$ is a function of $\left(\hat{\phi}, \hat{\rho}, x^{(i)}, y^{(i)}\right): \mathrm{B}^{(i)}\left(\hat{\phi}, \hat{\rho}, x^{(i)}, y^{(i)}\right)$. Fig. 1 shows an example of the error ellipses before and after the error covariance propagation.

Algorithm 1 gives a summary of the methods we propose to correct collinear points and update the corresponding error covariances.
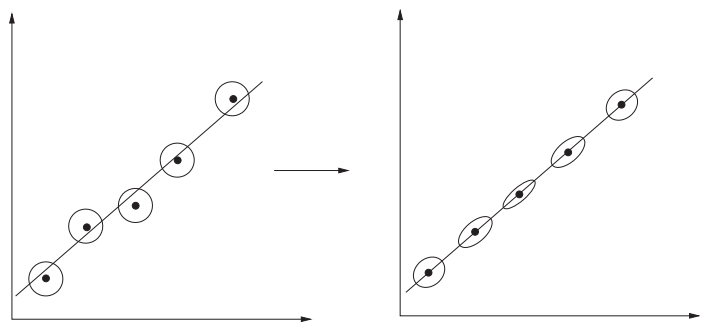

Fig. 1. Point error ellipses before and after projection.
Algorithm 1. Correcting collinear points and updating error covariances

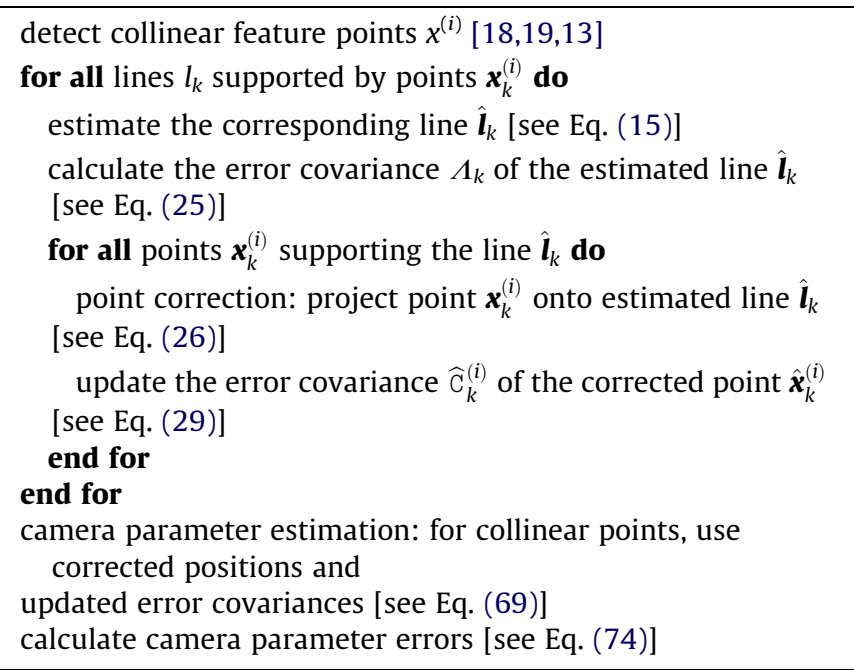

It can be intuitively verified that the error covariance component perpendicular to the line shows maximal decrease, whereas the component parallel to the line does not encounter any change. Furthermore, the error covariances are higher for the outer points on the line, compared with the points in the near vicinity of the centroid. However, these properties are observed only by experiments. In the following Sections, we derive these properties analytically from the Cramer-Rao bounds.

\section{Cramer-Rao bounds}

There are universal bounds for the accuracy of the estimated parameters determined by the Cramer-Rao bounds, so no estimator can yield parameter estimates which have lower error covariances than the Cramer-Rao bounds. In Section 4.1 the error covariance bounds for the line parameters are determined and in Section 4.3 the error covariance bounds for the projected point positions are specified. In Sections 4.2 and 4.4, obtained results are further analyzed and some fundamental properties are extracted.

\subsection{Cramer-Rao bounds for the error covariances of line parameters}

In order to calculate the lower bounds of the line parameter error covariances we need to determine the Fisher Information Matrix $\mathrm{F}^{l}$ which is defined as

$\mathrm{F}^{l}=-\mathrm{E}\left[\partial_{\phi, \rho} \partial_{\phi, \rho}^{\top} \ln [p(\boldsymbol{z} \mid \hat{\phi}, \hat{\rho})]\right]$,

where $\partial_{\phi, \rho} \partial_{\phi, \rho}^{\top}$ is the operator generating the Hessian and the superscript $l$ represents the line. The components of the Fisher Information Matrix are defined as

$\mathrm{F}_{m, n}^{l}=-\mathrm{E}\left[\partial_{m} \partial_{n} \ln [p(\boldsymbol{z} \mid \hat{\phi}, \hat{\rho})]\right]$,

with $m, n=1,2$ and $\partial_{1} \equiv \partial_{\phi}, \partial_{2} \equiv \partial_{\rho}$. Replacing $p(z \mid \hat{\phi}, \hat{\rho})$ yields

$$
\begin{aligned}
\mathrm{F}_{m, n}^{l} & =-\mathrm{E}\left[\partial_{m} \partial_{n} \ln \left[\prod_{i}^{M} \frac{e^{\left[-\frac{1}{2} \boldsymbol{d}^{(i)^{\top}} \mathrm{C}^{(i)^{-1}} \boldsymbol{d}^{(i)}\right]}}{2 \pi \sqrt{\operatorname{det}\left(\mathrm{C}^{(i)}\right)}}\right]\right], \\
& =\sum_{i}^{M} \frac{1}{2} \mathrm{E}\left[\partial_{m} \partial_{n} \boldsymbol{d}^{\left.(i)^{\top} \mathrm{C}^{(i)}{ }^{-1} \boldsymbol{d}^{(i)}\right],}\right. \\
& =\sum_{i}^{M} \frac{1}{2} \int\left\{\frac{e^{\left[-\frac{1}{2}\left(\hat{\boldsymbol{x}}-\boldsymbol{x}^{(i)}\right)^{\top} \mathrm{C}^{(i)^{-1}\left(\hat{\boldsymbol{x}}-\boldsymbol{x}^{(i)}\right)}\right]}}{2 \pi \sqrt{\operatorname{det}(\mathrm{C})}}\left[\partial_{m} \partial_{n} \boldsymbol{d}^{(i)^{\top}} \mathrm{C}^{(i)^{-1}} \boldsymbol{d}^{(i)}\right] \mathbf{d} \hat{\boldsymbol{x}}\right\} .
\end{aligned}
$$


The Cramer-Rao bounds are obtained by

$\operatorname{cov}(\hat{\phi}, \hat{\rho})_{m, n} \geqslant\left(\mathrm{~F}^{-1}\right)_{m, n}$.

As an example, in the case of isotropic covariance matrices for the point position error of the form

$\mathrm{c}^{(i)}=\left(\begin{array}{cc}\sigma^{2} & 0 \\ 0 & \sigma^{2}\end{array}\right)$

the components of the Fisher Information Matrix result in

$$
\begin{aligned}
\mathrm{F}_{1,1}^{l} & =-\sum_{i}^{M} \frac{2\left(y^{(i)}\right)^{2} \cos ^{2}(\phi)+x^{(i)} \cos (\phi) \rho}{\sigma^{2}} \\
& -\sum_{i}^{M} \frac{4 y^{(i)} x^{(i)} \cos (\phi) \sin (\phi)}{\sigma^{2}} \\
& -\sum_{i}^{M} \frac{\left(y^{(i)}\right)^{2}+y^{(i)} \sin (\phi) \rho}{\sigma^{2}} \\
& -\sum_{i}^{M} \frac{-2\left(x^{(i)}\right)^{2} \cos ^{2}(\phi)\left(x^{(i)}\right)^{2}}{\sigma^{2}} \\
\mathrm{~F}_{1,2}^{l} & =-\sum_{i}^{M} \frac{\sin (\phi) x^{(i)}-\cos (\phi) y^{(i)}}{\sigma^{2}} \\
\mathrm{~F}_{2,2}^{l} & =-\sum_{i}^{M} \frac{1}{\sigma^{2}} .
\end{aligned}
$$

4.2. Interpretation of Cramer-Rao bounds for the error covariances of line parameters

In order to achieve more insight into the relationships between the number of points, their accuracy and the accuracy of the estimated line, we further analyze the terms for the Cramer-Rao bounds. Without loss of generality, we assume that the estimated line lies on the $x$-axis:

$\hat{\phi}=\frac{\pi}{2}, \quad \hat{\rho}=0$.

By using Eqs. (38) and (37), we get

$\mathrm{F}_{1,1}^{l}=\sum_{i}^{M} \frac{-\left(\hat{y}^{(i)}\right)^{2}+\left(\hat{\boldsymbol{x}}^{(i)}\right)^{2}}{\sigma^{2}}$,

$\mathrm{F}_{1,2}^{l}=\mathrm{F}_{2,1}^{l}=\sum_{i}^{M} \frac{\hat{x}^{(i)}}{\sigma^{2}}$,

$\mathrm{F}_{2,2}^{l}=\frac{M}{\sigma^{2}}$

For the Fisher Information Matrix follows:

$\mathrm{F}^{l}=\frac{1}{\sigma^{2}}\left(\begin{array}{cc}\sum_{i}^{M}-\left(\hat{y}^{(i)}\right)^{2}+\left(\hat{\boldsymbol{x}}^{(i)}\right)^{2} & \sum_{i}^{M} \hat{\boldsymbol{x}}^{(i)} \\ \sum_{i}^{M} \hat{\boldsymbol{x}}^{(i)} & M\end{array}\right)$.

Determining the inverse matrix $\mathrm{F}^{l-1}$ yields

$\mathrm{F}^{l^{-1}}=v \cdot\left(\begin{array}{cc}M & -\sum_{i}^{M} \hat{\boldsymbol{x}}^{(i)} \\ -\sum_{i}^{M} \hat{\boldsymbol{x}}^{(i)} & \sum_{i}^{M}-\left(\hat{y}^{(i)}\right)^{2}+\left(\hat{\boldsymbol{x}}^{(i)}\right)^{2}\end{array}\right)$,

where

$v=\frac{\sigma^{2}}{M \cdot\left(\sum_{i}^{M}-\left(\hat{y}^{(i)}\right)^{2}+\left(\hat{x}^{(i)}\right)^{2}\right)-\left(\sum_{i}^{M} \hat{x}^{(i)}\right)^{2}}$.

To further simplify the terms, we assume that each point has the same distance $a$ to its neighbor and that $\hat{x}^{(1)}=a$. This means $\hat{x}^{(i)}=a \cdot i, \quad \hat{y}^{(i)}=0, \quad i=1, \ldots, M$.

This assumption leads to the following Fisher Information Matrix

$$
\mathrm{F}^{l^{-1}}=\frac{\sigma^{2}}{M S_{1}-S_{2}}\left(\begin{array}{cc}
M & -S_{3} \\
-S_{3} & S_{1}
\end{array}\right),
$$

where $S_{1}, S_{2}$ and $S_{3}$ are given by

$$
\begin{aligned}
& S_{1}=\sum_{i}^{M}\left(\hat{x}^{(i)}\right)^{2}=\sum_{i}^{M} a^{2} i^{2}=\left(\frac{1}{3} a^{2} M^{3}+\frac{1}{2} a^{2} M^{2}+\frac{1}{6} a^{2} M\right) \\
& S_{2}=\left(\sum_{i}^{M} \hat{x}^{(i)}\right)^{2}=\left(\sum_{i}^{M} a i\right)^{2}=\frac{1}{4} a^{2}(M+1)^{2} M^{2} \\
& S_{3}=\sum_{i}^{M} \hat{x}^{(i)}=\sum_{i}^{M} a i=\frac{1}{2} a M^{2}+\frac{1}{2} a M .
\end{aligned}
$$

By defining $S_{4}$ as

$S_{4}=M S_{1}-S_{2}=\frac{1}{12} a^{2}\left(M^{4}-M^{2}\right)$,

and using the following identities

$\frac{S_{3}}{S_{4}}=\frac{6}{a M(M-1)}, \frac{S_{1}}{S_{4}}=\frac{2(2 M+1)}{M(M-1)}$,

we get the final simplified lower bounds for the errors of $\hat{\phi}, \hat{\rho}$, respectively, from (46):

$$
\mathrm{F}^{l^{-1}}=\sigma^{2}\left(\begin{array}{cc}
\frac{12}{M a^{2}\left(M^{2}-1\right)} & -\frac{6}{a M(M-1)} \\
-\frac{6}{a M(M-1)} & \frac{4 M+2}{M(M-1)}
\end{array}\right) .
$$

Following results can be deduced from Eq. (52). The error of the line parameters $\hat{\phi}, \hat{\rho}$ is unbiased, because

$$
\lim _{M \rightarrow \infty} \mathrm{F}^{l^{-1}}=\left(\begin{array}{ll}
0 & 0 \\
0 & 0
\end{array}\right)
$$

Furthermore, the error variance of $\hat{\phi}$ is proportional to $\frac{1}{a^{2}}$, i.e. greater distance $a$ leads to smaller error of $\hat{\phi}$. In contrast, the error of $\hat{\rho}$ does not depend on the distance between the supporting points. This means that widening the supporting point set only increases the accuracy of the angle, but not the accuracy of the distance to the origin of the coordinate system. Both parameters of the estimated line become more accurate with increasing number $M$ of supporting points.

\subsection{Cramer-Rao bounds for the error covariances of corrected point positions}

The projection mapping (26) is a function of $(\phi, \rho, x, y): \boldsymbol{P}(\phi, \rho, x$, $y)$. Since the Cramer-Rao bounds for $\hat{\phi}, \hat{\rho}$ are already determined and the true PDF's of $x^{(i)}$ are assumed to be known, Eq. (3.30) from [21] is used in order to obtain the inverse Fisher Information Matrix $\left({ }_{F} \hat{p}^{(i)}\right)^{-1}$ regarding the lower bounds of the covariances of the corrected points:

$$
\left({ }_{F} \hat{\boldsymbol{p}}^{(i)}\right)^{-1}=\left[\partial_{\theta} \boldsymbol{P}(\boldsymbol{\theta})\right] \mathrm{F}^{-1}(\boldsymbol{\theta})\left[\partial_{\theta} \boldsymbol{P}(\boldsymbol{\theta})\right]^{\top}
$$

where $\boldsymbol{\theta}=(\hat{\phi}, \hat{\rho}, x, y)^{\top}$. The term $\mathrm{F}^{-1}(\boldsymbol{\theta})$ represents the inverse Fisher Information Matrix containing the Cramer-Rao bounds for the 
estimated line parameters $\hat{\phi}, \hat{\rho}$ and the feature point coordinates $x$, $y$. The Fisher Information Matrix $\mathrm{F}(\boldsymbol{\theta})$ is defined as

$\mathrm{F}(\boldsymbol{\theta})=\left(\begin{array}{cccc}\mathrm{F}_{1,1}^{l} & \mathrm{~F}_{1,2}^{l} & 0 & 0 \\ \mathrm{~F}_{2,1}^{l} & \mathrm{~F}_{2,2}^{l} & 0 & 0 \\ 0 & 0 & \left(\mathrm{C}^{(i)^{-1}}\right)_{1,1} & \left(\mathrm{C}^{(i)^{-1}}\right)_{1,2} \\ 0 & 0 & \left(\mathrm{C}^{(i)}\right)_{2,1} & \left(\mathrm{C}^{(i)}\right)_{2,2}^{-1}\end{array}\right)$.

Since the term $\partial_{\boldsymbol{\theta}} \boldsymbol{P}(\boldsymbol{\theta})$ is already defined in (27) as B, it may be written

$\left(\mathrm{F}^{\hat{p}^{(i)}}\right)^{-1}=\mathrm{B}(\boldsymbol{\theta}) \mathrm{F}^{-1}(\boldsymbol{\theta}) \mathrm{B}(\boldsymbol{\theta})^{\top}$.

4.4. Interpretation of Cramer-Rao bounds for the error covariances of corrected point positions

For further analysis of the position error of a corrected feature point $\hat{\boldsymbol{x}}^{(i)}$, we start at the corresponding covariance matrix from Eq. (29). Since the Jacobian $\mathrm{B}^{(i)}$ does not depend on the error covariances, the lower bound $\left({ }_{\mathrm{F}} \hat{\boldsymbol{p}}^{(i)}\right)^{-1}$ for $\widehat{\mathrm{C}}^{(i)}$ is determined by

$$
\left(\mathrm{F}^{\hat{p}^{(i)}}\right)^{-1}=\mathrm{B}^{(i)}\left(\begin{array}{cccc}
\mathrm{F}_{1,1}^{l} & \mathrm{~F}_{1,2}^{l} & 0 & 0 \\
\mathrm{~F}_{2,1}^{l} & \mathrm{~F}_{2,2}^{l} & 0 & 0 \\
0 & 0 & \left(\mathrm{C}^{(i)^{-1}}\right)_{1,1} & \left(\mathrm{C}^{(i)^{-1}}\right)_{1,2} \\
0 & 0 & \left(\mathrm{C}^{(i)^{-1}}\right)_{2,1} & \left(\mathrm{C}^{(i)^{-1}}\right)_{2,2}
\end{array}\right)^{-1} \mathrm{~B}^{(i)^{\top}} .
$$

With the assumption of isotropic covariance matrices, as indicated by Eq. (36), it follows that

$$
\left(\mathrm{F}^{\hat{p}^{(i)}}\right)^{-1}=\mathrm{B}^{(i)}\left(\begin{array}{cccc}
\mathrm{F}_{1,1}^{l} & \mathrm{~F}_{1,2}^{l} & 0 & 0 \\
\mathrm{~F}_{2,1}^{l} & \mathrm{~F}_{2,2}^{l} & 0 & 0 \\
0 & 0 & \frac{1}{\sigma^{2}} & 0 \\
0 & 0 & 0 & \frac{1}{\sigma^{2}}
\end{array}\right)^{-1} \quad \mathrm{~B}^{(i)^{\top}} .
$$

Again, we assume that the estimated line lies on the $x$-axis and the points are equidistant:

$x^{(i)}=a \cdot i, \quad y^{(i)}=0, \quad i=1, \ldots, M$.

With the additional weak assumption

$$
a \gg \sigma
$$

and the use of Eqs. (52) and (58) follows:

$$
\left(\mathrm{F}^{\hat{\boldsymbol{p}}^{(i)}}\right)^{-1}=\sigma^{2}\left(\begin{array}{cc}
1 & 0 \\
0 & \frac{2+4 M^{2}-12 M i+6 M+12 i^{2}-12 i}{M^{3}-M}
\end{array}\right) .
$$

One result from Eq. (61) is that the covariance component corresponding to the direction of the estimated line is unchanged by the correction:

$$
\left(\mathrm{F}^{\hat{\boldsymbol{p}}^{(i)}-1}\right)_{1,1}=\mathrm{C}_{1,1}^{(i)}=\sigma^{2} .
$$

On the other hand, the $y$-component $\left({ }_{F} \hat{p}^{(i)}-1\right)_{2,2}$ is decreased. In order to determine which corrected feature point has the least error, we determine the $i$-derivative of $\left(\mathrm{F}^{\hat{\boldsymbol{p}}^{(i)}-1}\right)_{2,2}$, which is supposed to yield zero. For a constant $M$, the denominator may be discarded. The numerator is a 2-nd degree polynomial in $i$

$$
\begin{aligned}
\partial_{i} & \left(2+4 M^{2}-12 M i+6 M+12 i^{2}-12 i\right)=-12 M+24 i-12 \\
\quad & 0 \Rightarrow i=\frac{M+1}{2} .
\end{aligned}
$$

From this follows that points with $i=\frac{M+1}{2}$ in the vicinity of the centroid

$$
\left(\begin{array}{c}
a \frac{M+1}{2} \\
0
\end{array}\right)
$$

of supporting points have smallest error variance in the $y$-component. The error variance increases with the distance to the centroid symmetrically, since it is

$\left(\mathrm{F}^{\hat{p}^{(j)}-1}\right)_{2,2}=\left(\mathrm{F}^{\hat{p}^{(M-j+1)}-1}\right)_{2,2}$.

This is simply shown by the substitution of $i=M-j+1$ in $\left(\mathrm{F}^{\hat{\boldsymbol{p}}^{(i)}-1}\right)_{2,2}$

$$
\begin{aligned}
&\left(\mathrm{F}^{\hat{\boldsymbol{p}}^{(M-j+1)}-1}\right)_{2,2}=2+4 M^{2}-12 M(M-j+1)+6 M \\
&+12(M-j+1)^{2}-12(M-j+1) \\
&= 2+4 M^{2}-12 M j+6 M+12 j^{2}-12 j \\
&=\left(\mathrm{F}^{\hat{p}^{(j)}-1}\right)_{2,2} .
\end{aligned}
$$

In an example with 5 points, Fig. 2 shows the symmetric distribution of the variances of the $y$-component.

One expects no improvement of the accuracy in case of only $M=2$ supporting points. This is shown by

$\mathrm{F}^{\hat{p}^{(1)-1}}=\mathrm{F}^{\hat{p}^{(2)-1}}=\sigma^{2}\left(\begin{array}{ll}1 & 0 \\ 0 & 1\end{array}\right)$.

It is also easily shown that for the case $M>2$, the accuracy of all points improves. We simply have to test for

$\frac{2+4 M^{2}-12 M i+6 M+12 i^{2}-12 i}{M^{3}-M}<1$,

which is true for all $M>2$.

\section{Maximum-Likelihood estimation and covariance propagation of camera parameters}

ML-estimation of camera parameters is performed by the bundle adjustment method [12], in which the 3D-feature points and the camera parameters are estimated simultaneously. In order to determine the error covariances, a brief review of the basic principles of the estimation process are given.

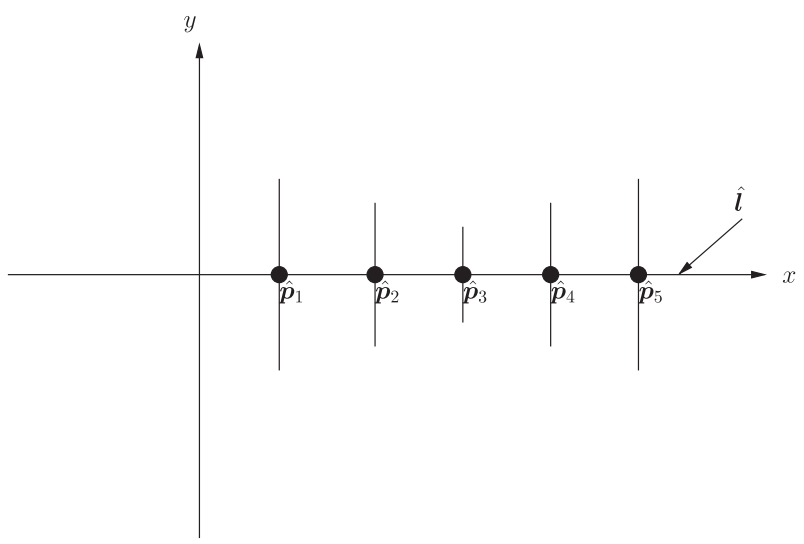

Fig. 2. Distribution of error variances of the $y$-component. The vertical lines indicate the corresponding variances. 

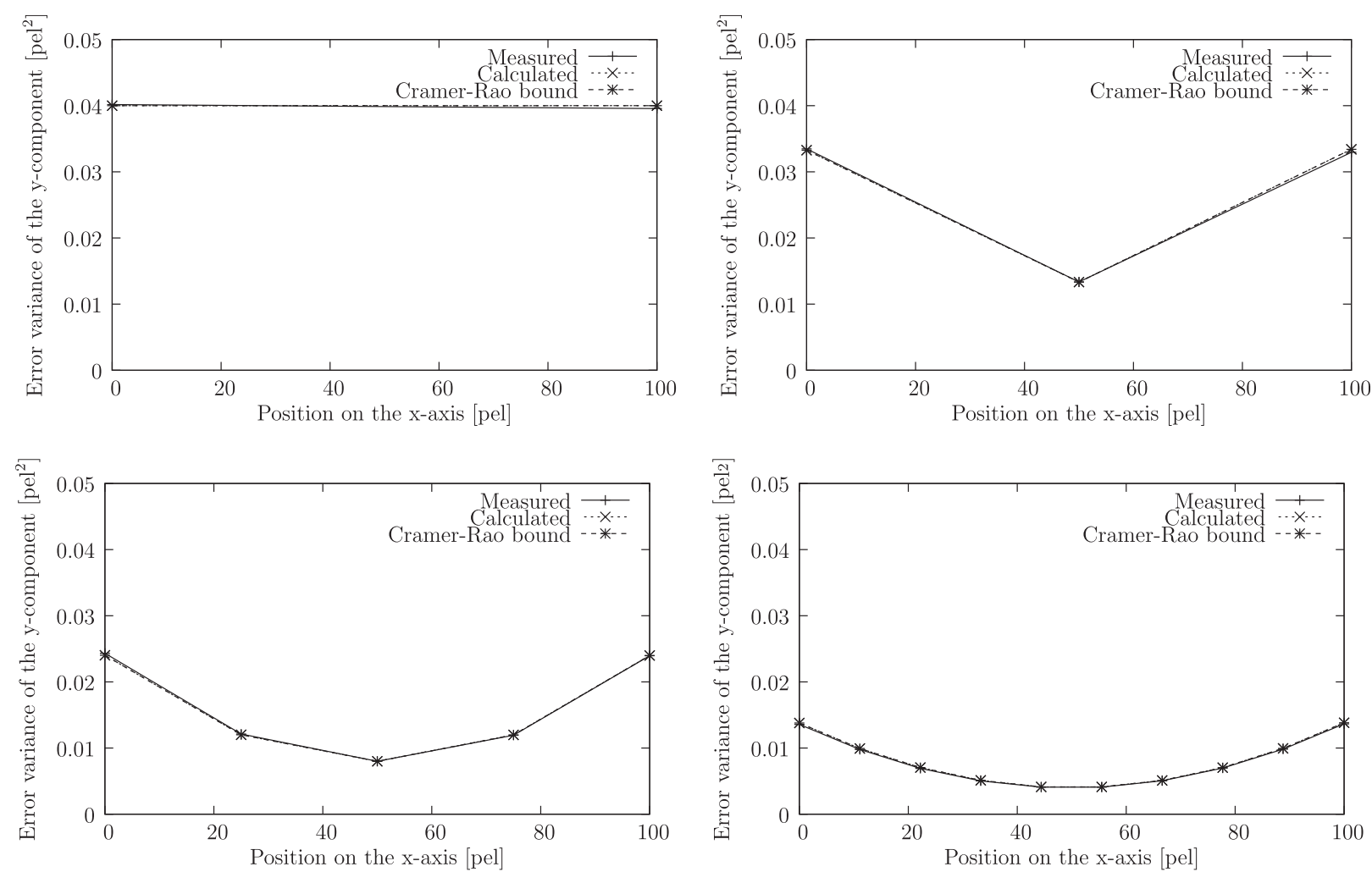

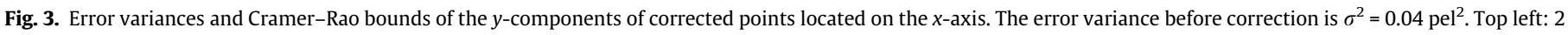
collinear points, top right: three collinear points, bottom left: five collinear points and bottom right: 10 collinear points.

Let there be $V$ views and $N$ 3D-points. The ML-estimation is then defined by

$\widehat{\boldsymbol{Q}}=\arg \min _{\mathbf{Q}} \sum_{i}^{V} \sum_{j}^{N} d\left(\boldsymbol{x}^{(i, j)}, \mathrm{P}\left(\boldsymbol{q}^{(i)}, \boldsymbol{X}^{(j)}\right)\right)_{\mathrm{C}^{(i, j)}}^{2}$,

where $\boldsymbol{x}^{(i, j)}$ is the $j$ th 2D-point of the $i$ th view, P is the projection function, $\boldsymbol{q}^{(i)}$ is the vector containing $r$ camera parameters, $\boldsymbol{X}^{(j)}=\left(X_{1}^{(j)}, X_{2}^{(j)}, X_{3}^{(j)}\right)^{\top}$ is the $j$ th 3D-point, $\boldsymbol{Q}$ the vector representing all parameters to be estimated

$\boldsymbol{Q}=\left(q_{1}^{(1)}, \ldots, q_{r}^{(V)}, X_{1}^{(1)}, \ldots, X_{3}^{(N)}\right)^{\top}$,

and $d(\ldots)_{\mathbb{C}^{(i, j)}}$ is the Mahalanobis distance according to the covariance matrix $\mathrm{C}^{(i, j)}$. Let $f(\widehat{\boldsymbol{Q}})$ be the function projecting all estimated 3D points onto the camera plane

$$
\begin{aligned}
f(\widehat{\boldsymbol{Q}}) & =\left(\mathrm{P}\left(\hat{\boldsymbol{q}}^{(1)}, \hat{\boldsymbol{X}}^{(1)}\right), \ldots, \mathrm{P}\left(\hat{\boldsymbol{q}}^{(V)}, \hat{\boldsymbol{X}}^{(N)}\right)\right)^{\top} \\
& =\left(\hat{\boldsymbol{\xi}}^{(1,1)}, \ldots, \hat{\boldsymbol{\xi}}^{(V, N)}\right)^{\top} .
\end{aligned}
$$

By collecting the covariance matrices as

$\Sigma=\left(\begin{array}{ccc}\mathrm{C}^{(1,1)} & & \\ & \ddots & \\ & & \mathrm{C}^{(V, N)}\end{array}\right)$,

the covariance of the estimated parameters is obtained [12] by

$\operatorname{cov}(\widehat{\mathbf{Q}})=\left(J^{\top} \Sigma^{-1} J\right)^{-1}$,

with the Jacobian

$J=\left.\frac{d f}{d \mathbf{Q}}\right|_{\mathbf{Q}=\hat{\mathbf{Q}}}$.
In order to determine the covariance in presence of collinear points, each collinear point $\boldsymbol{x}^{(i, j)}$ is replaced by its corrected point $\hat{\boldsymbol{x}}^{(i, j)}$ and each corresponding covariance matrix $C^{(i, j)}$ is replaced by the covariance matrix $\widehat{\mathrm{C}}^{(i, j)}$, as determined in (29).

\section{Experimental results}

To demonstrate the impact of collinear features on the expected analytic covariances, camera parameter estimation is performed utilizing simulated data as well as real data image sequence. It is assumed that the estimated parameter errors are unbiased, so that they have zero means. The estimation of camera parameter errors is done by the method presented in Section 5 .

\subsection{Simulated data tests}

First, the error analysis is verified by simulation experiments based on 2D-points. Fig. 3 shows the results for varying number

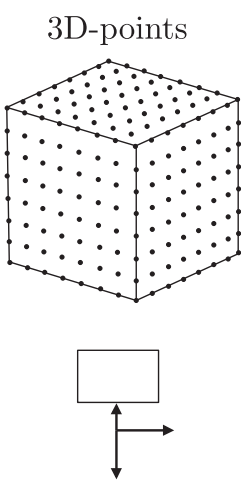

Camera view 2

Fig. 4. Synthetic camera and 3D-point setup. 

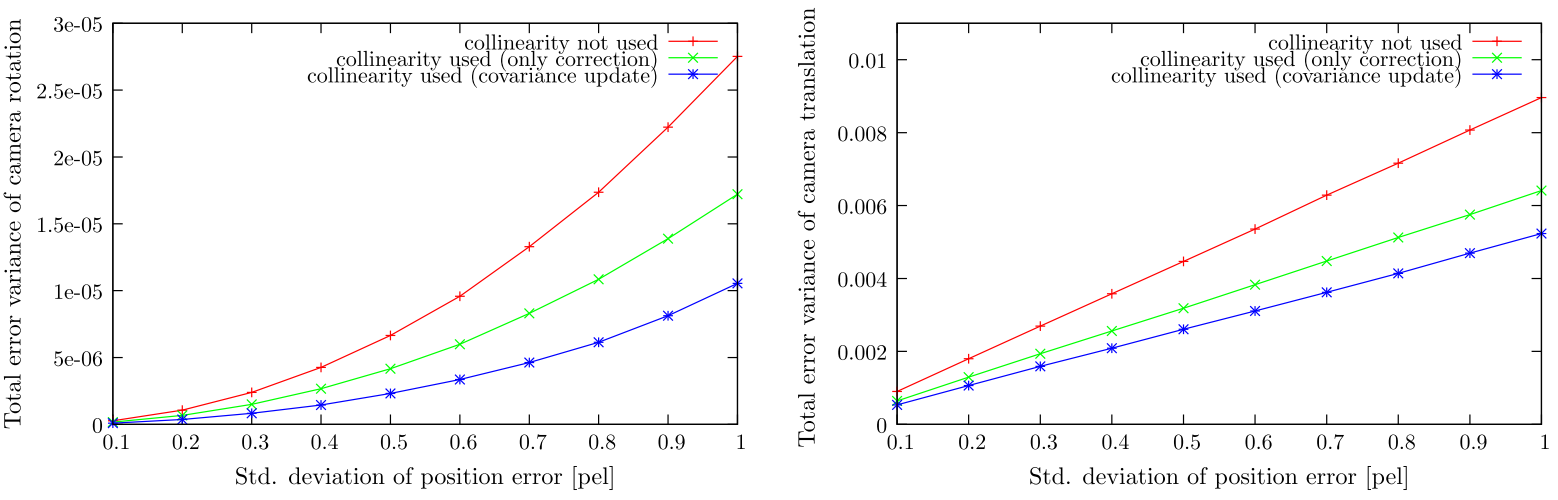

Fig. 5. Rotation angle error variance (left) and normalized translation error variance.
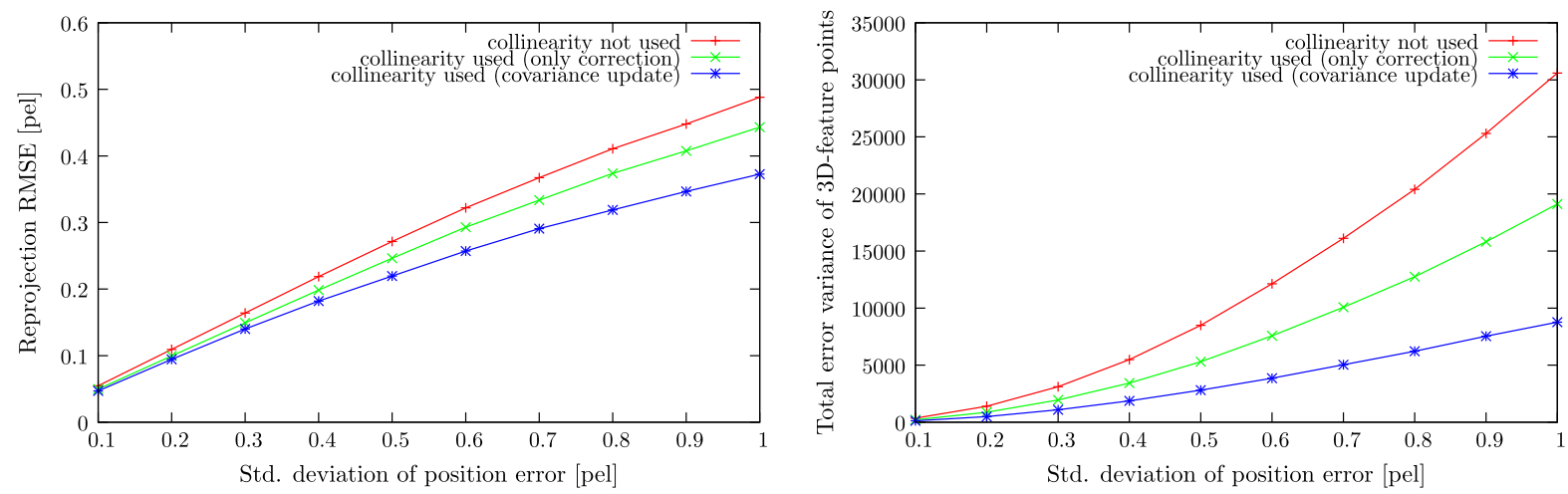

Fig. 6. Reprojection RMSE (left) and 3D-reconstruction error variance.
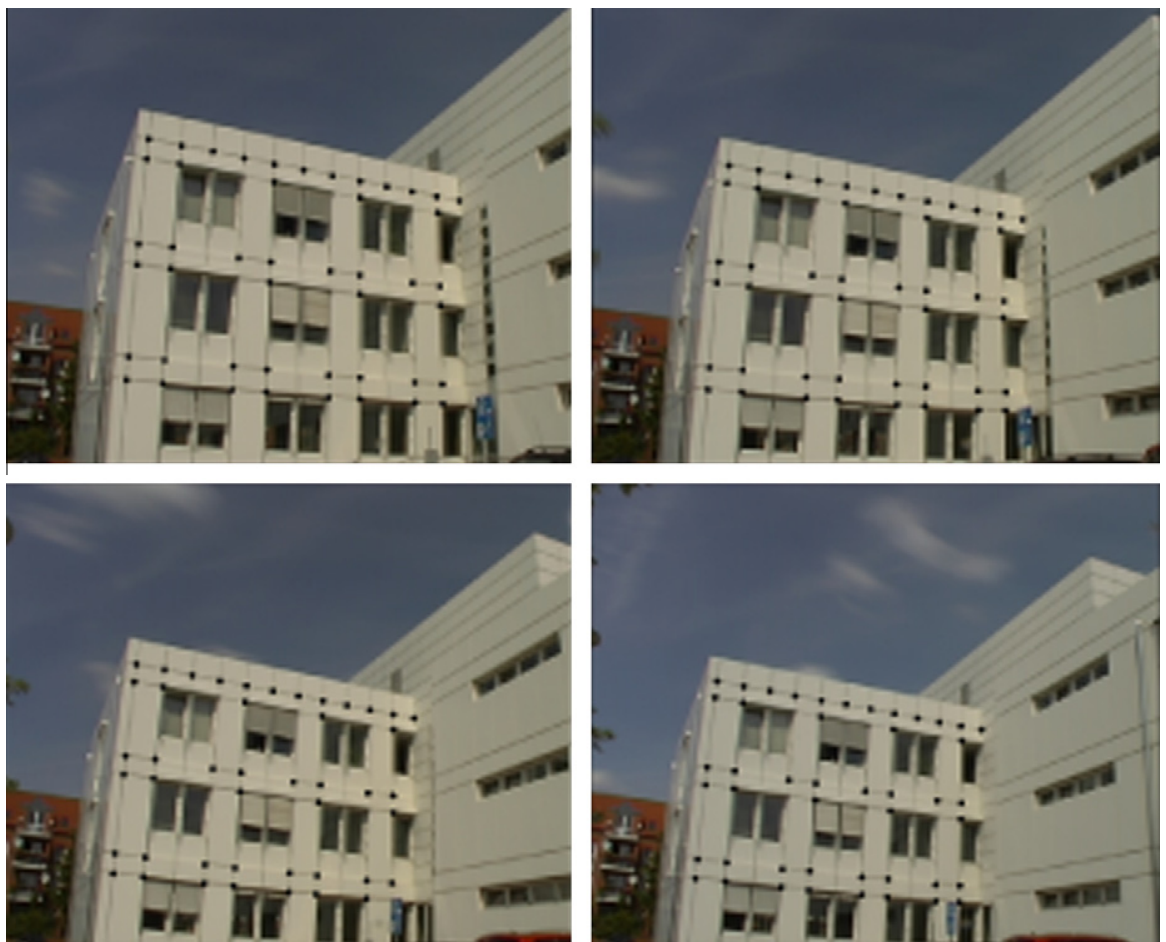

Fig. 7. Real data image sequence: images (numbers 1, 3, 5 and 7) with detected collinear feature points.

of simulated collinear points located on the $x$-axis. The application of the error analysis is compared to statistical measurements, showing a very good conformance. The measured error variances are obtained by 100 simulations.
As predicted, the minimum error variance is found in the vicinity of the centroid. For example, with five collinear points, the error variance of the corrected point in the vicinity of the centroid decreases by a factor of $1 / 5$. Also, the dependency on 

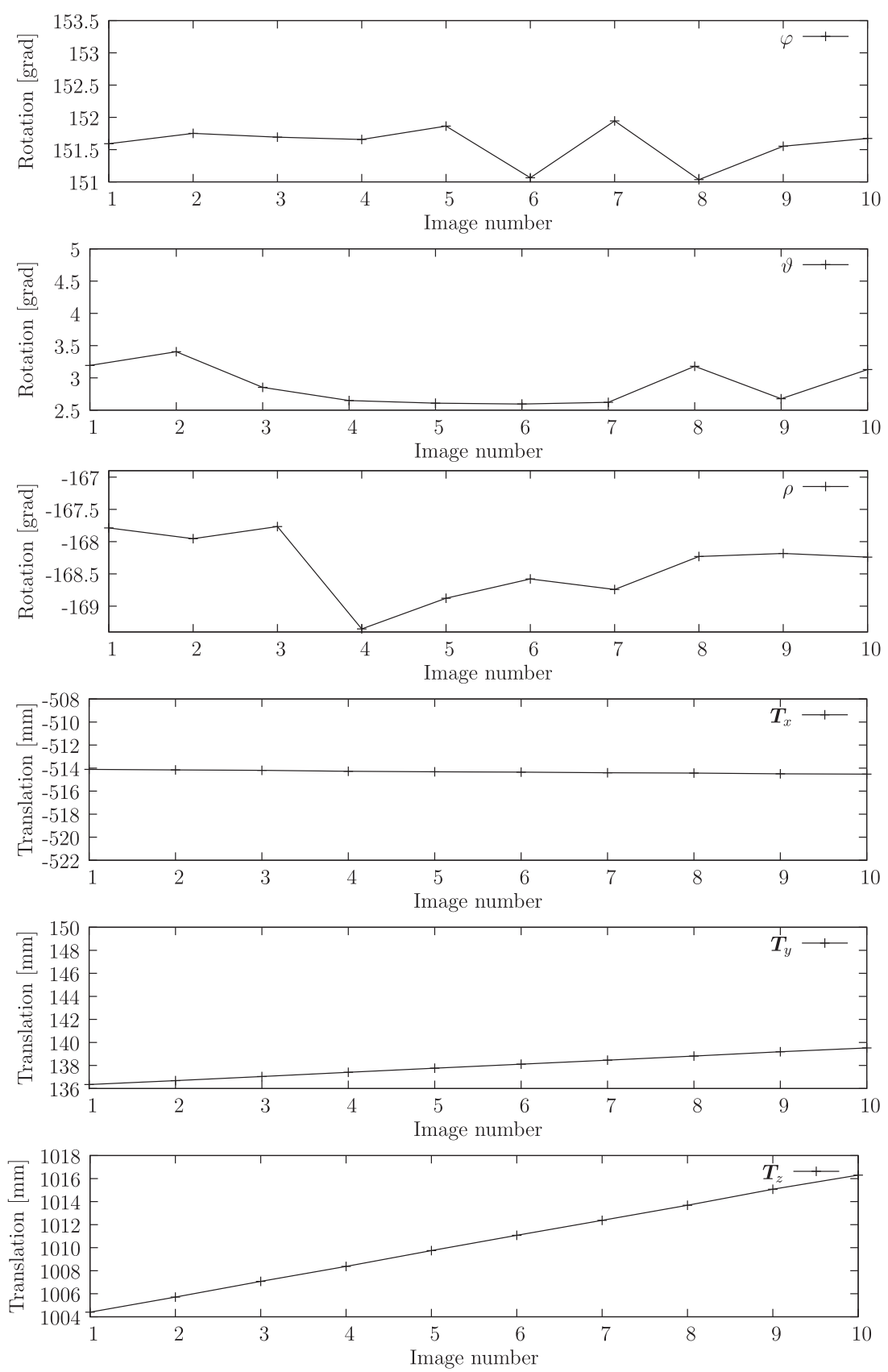

Fig. 8. Estimated camera parameters corresponding to the noise-free real data image sequence.

the number of collinear points is demonstrated by an overall decrease.

The second part of simulated data based experiments consists of regularly positioned 3D-points within a cube, as shown in Fig. 4. There are 12 line segments detected, vertical ones as well as horizontal ones. Therefore, the error of corrected points shows a consecutive decrease of covariance in both directions. Experiments are done with increasing standard deviations for the position error of the 2D-points, which are determined in two camera views. Each plot shows three curves for the parameter error: one without exploiting the collinearity of the 2D-points, a second for exploiting collinearity by only correcting points and a third curve for correcting points with full covariance update. Fig. 5 shows the results for the error variance of camera rotation and normalized translation, respectively. The normalized translation error variance is calculated from the translation unit vector. Fig. 6 shows the reprojection RMSE and the 3D-reconstruction error variance, respectively.

In all cases, exploiting the collinearity results in a considerable decrease in the error of camera parameters. Updating the error covariances decreases the errors even more.

\subsection{Real data tests}

The real data based experiment consists of $10 \times 100$ images. There are 10 camera positions and on each position, 100 images are taken. The focal length is $f=6.85 \mathrm{~mm}$. Using the 100 images per camera position, a mean image is calculated. Since a zero mean Gaussian error for the pixel intensities is assumed, the mean image is supposed to be approximately noise-free. The noise-free image is used to determine the PSNR of the noisy images. Using all 10 noise-free images, the ground truth camera 


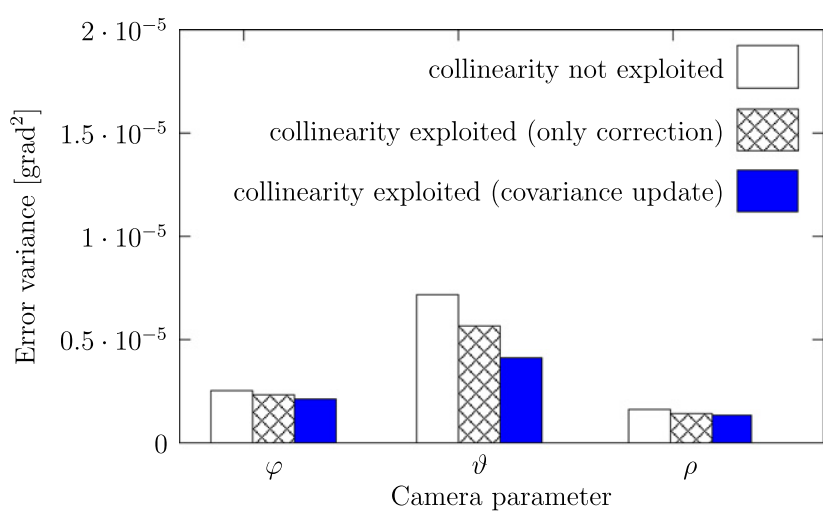

Fig. 9. Measured error variance of estimated camera rotation parameters. The first estimation does not exploit collinearity. The second estimation is done by correcting collinear feature points. The third estimation is done by correcting and updating the corresponding error covariances. PSNR $=38.4 \mathrm{~dB}, \sigma=0.2$ pel.

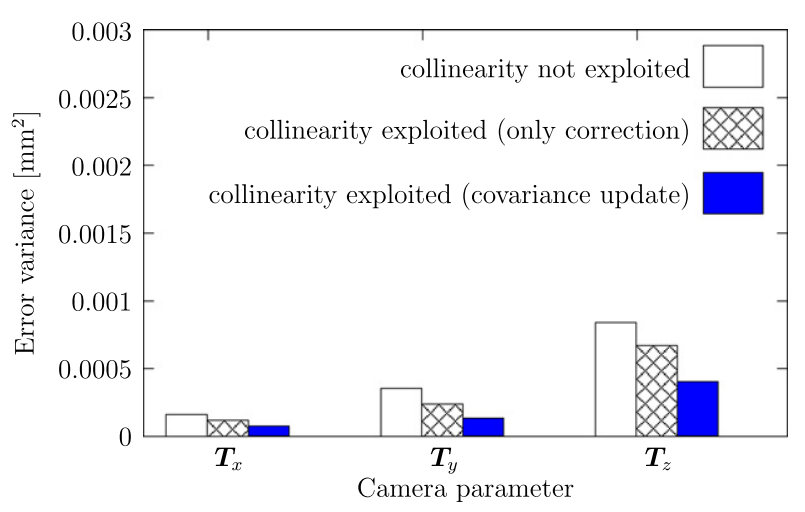

Fig. 10. Measured error variance of estimated camera translation parameters. The first estimation does not exploit collinearity. The second estimation is done by correcting collinear feature points. The third estimation is done by correcting and updating the corresponding error covariances. PSNR $=38.4 \mathrm{~dB}, \sigma=0.2$ pel.

parameters are calculated. Using one noisy image per camera position, the camera parameter errors are determined.

Because of many collinear features in the images this image sequence is well suited to test the improvement of the accuracy by exploiting the collinearity. Fig. 7 shows some images from the sequence. Fig. 8 shows the estimated camera parameters. The camera motion consists mainly of a forward translation and small rotational motions due to manhandling.

Figs. 9 and 10 show the measured error variance of the camera rotation and camera translation parameters, respectively. The error variance of the rotation angle $\vartheta$ is decreased by $20 \%$ (correction only) and 40\% (correction + covariance update). Error variances of the other rotation angles show no significant change. The error variances of the translation parameters are decreased by $15-30 \%$ (correction only) and 40-60\% (correction + covariance update). In average, correction with covariance update decreases the error variance of the rotation parameters by approximately 30\%. Error variance of translation parameters is decreased by approximately $50 \%$ in average.

\section{Conclusions}

In this paper an algebraic error covariance propagation for camera parameter estimation in presence of collinear feature points is presented. The ML-estimation of the supporting lines and the cor- responding covariance propagation is reviewed. Furthermore, the correction of collinear points and the corresponding covariances is determined.

By determining the Fisher information matrix the lower bounds for the error covariance of the corrected point positions are obtained. Further analysis of the Cramer-Rao bounds reveal fundamental properties of the relationship of error covariances, the number of supporting points and their distance. It is shown that the maximal gain in accuracy by correcting collinear points is encountered in the vicinity of their centroid. Furthermore, it is shown that the error covariance component perpendicular to the supporting line shows maximal decrease whereas the component parallel to the supporting line does not encounter any change.

Finally, algebraic as wall as experimental investigations show how much the accuracy of camera parameters can be increased by taking advantage of the information about the collinearity of feature points.

\section{Acknowledgment}

This work was partly published in IEEE Proceedings of the Canadian Conference on Computer and Robot Vision 2006 (CRV2006).

\section{References}

[1] Q.-T. Luong, O.D. Faugeras, Self-calibration of a camera using multiple images, in: 11th IAPR International Conference on Pattern Recognition, vol. 1, 1992, pp. 9-12.

[2] Q.-T. Luong, R. Deriche, O. Faugeras, T. Papadopoulo, On determining the fundamental matrix: analysis of different methods and experimental results, 1993, Inria. Available from: <http://www.inria.fr/rapports/sophia/RR-1894. html>

[3] Q.-T. Luong, O. Faugeras, The fundamental matrix: theory, algorithms, and stability analysis, International Journal of Computer Vision 17 (1) (1996) 4376.

[4] P.H.S. Torr, A. Zisserman, S.J. Maybank, Robust detection of degenerate configurations for the fundamental matrix, in: IEEE International Conference on Computer Vision, 1995, pp. 1037-1042.

[5] P.H.S. Torr, Motion Segmentation and Outlier Detection, Dissertation, University of Oxford, 1995.

[6] P.H.S. Torr, D. Murray, The development and comparison of robust methods for estimating the fundamental matrix, International Journal of Computer Vision 24 (3) (1997) 271-300.

[7] P.H.S. Torr, A. Zisserman, Robust parameterization and computation of the trifocal tensor, Image and Vision Computing 15 (3) (1997) 591-605.

[8] R.I. Hartley, In defense of the eight-point algorithm, in: IEEE International Conference on Computer Vision, 1995, pp. 1064-1070.

[9] R.I. Hartley, Self-calibration of stationary cameras, International Journal of Computer Vision 22 (1) (1997) 5-23.

[10] R.I. Hartley, Lines and points in three views and the trifocal tensor, International Journal of Computer Vision 22 (2) (1997) 125-140.

[11] R.I. Hartley, Minimizing algebraic error in geometric estimation problems, in: IEEE International Conference on Computer Vision, 1998, pp. 469-476.

[12] R.I. Hartley, A. Zisserman, Multiple View Geometry, Cambridge University Press, 2000.

[13] A. Bartoli, M. Coquerelle, P. Sturm, A framework for pencil-of-points structurefrom-motion, in: European Conference on Computer Vision, vol. 2, Springer, 2004, pp. 28-40.

[14] G. Liu, R. Klette, B. Rosenhahn, Structure from motion in the presence of noise, in: Image and Vision Computing New Zealand, 2005.

[15] D. Forsyth, J. Ponce, Computer Vision: A Modern Approach, Prentice Hall, Upper Saddle River, New Jersey, 2000.

[16] G. Speyer, M. Werman, Parameter estimates for a pencil of lines: Bounds and estimators, in: European Conference on Computer Vision, Springer, Copenhagen, 2002, pp. 432-446.

[17] R. Duda, P. Hart, Pattern Classification and Scene Analysis, John Wiley \& Sons, 1973.

[18] C. Tomasi, T. Kanade, Detection and Tracking of Point Features, Carnegie Mellon University Technical Report CMU-CS-91-132, April 1991.

[19] J. Shi, C. Tomasi, Good features to track, in: IEEE Conference on Computer Vision and Pattern Recognition, 1994, pp. 593-600.

[20] R. Szeliski, Bayesian Modeling of Uncertainty in Low-level Vision, Kluwer Academic Publishers, Boston, 1989.

[21] S.M. Kay, Fundamentals of Statistical Signal Processing, Estimation Theory, vol. I, Prentice Hall, Upper Saddle River, 1993. 